
\title{
BReserch S Suare \\ Further placental pathology in mild COVID-19 of term pregnancy with pathophysiological correlates
}

Anastasia E. Konstantinidou ( $\sim$ ankon@med.uoa.gr)

Vassiliki Papaevangelou

Athanasios Tsakris

Nikolaos E. Spanakis

Garyfallia Syridou

Alexandros Pergaris

Eleni Kapsabeli

Vassiliki Sideri

Anna Daskalaki

Sophia N. Kalantaridou

\section{Research Article}

Keywords: SARS-COV-2, placenta, inflammatory lesions, endothelial vacuolation, thrombosis, fibrin degradation

Posted Date: July 8th, 2020

DOl: https://doi.org/10.21203/rs.3.rs-40375/v1

License: (c) (1) This work is licensed under a Creative Commons Attribution 4.0 International License. Read Full License 


\section{Abstract}

Placental pathology related to SARS-COV-2 infection during pregnancy is under investigation, with emerging but still limited and variable data, mainly including changes of fetal and maternal vascular malperfusion. We observed novel pathological findings not previously reported in two term placentas delivered from women mildly affected with COVID-19, with a 2-day and 6-day interval between the onset of symptoms and delivery. The main changes involved the maternal more than the fetal components of the placenta and included a tendency toward thrombosis and fibrin formation, and mild maternal malperfusion. We further observed fibrin degeneration in the intervillous space, the presence of particles of undetermined origin, and mild or moderate maternal decidual inflammatory exudates consisting of monocytes, macrophages, T-lymphocytes, and plasmacytes. Mild inflammation and incipient mural thrombus formation were noted in the fetal vasculature, as well as endothelial vacuolation of the umbilical vessels, not previously described. The maternal changes were more prominent in the case with a relatively longer clinical manifestation-to-delivery interval, and were associated to a prolonged postpartum maternal viral carriage of 30 days and the development of a low-grade febrile response in the neonate. Both infants showed mild morbidity but eventually had a very good outcome. RT-PCR was negative for SARS-CoV-2 RNA in both cases.

We concluded that placental involvement in mild COVID-19 of very short duration at term pregnancy suggested the systemic nature of the disease and appeared related to, though not pathognomonic of COVID-19. Fetoplacental vascular changes and endothelial vacuolation associated with mild neonatal morbidity may suggest a possible transplacental impact on the fetus, to be further investigated.

\section{Introduction}

The current coronavirus disease 2019 (COVID-19) pandemic, caused by the severe acute respiratory syndrome coronavirus 2 (SARS-CoV-2) has raised, among many others, major concerns regarding the impact of infection during pregnancy. As of the time of this writing vertical SARS-COV-2 transmission remains controversial, while viral RNA has been detected in some placentas of COVID-19-affected mothers suggesting placental infection [1-3]. Emerging data on COVID-19-induced placental pathology are still limited $[4,5]$. We herein present in detail the placental pathology in two cases of pregnant women mildly affected with COVID-19 at term, and describe the pathological changes, including novel findings not previously reported, along with their pathophysiological correlates.

\section{Materials And Methods}

Two placentas delivered from pregnant women with confirmed COVID-19 infection were sent for pathological examination to the 1rst University Department of Pathology, Unit of Perinatal Pathology, National Kapodistrian University of Athens (NKUA). The placentas were delivered to the Pathology 
department immersed in $10 \%$ formalin and were fixed for 4 days before initial examination with personal protective equipment. The specimens were then trimmed of the free membranes and cord, photographed, weighed, sampled for PCR-testing, and re-immersed in formalin for 7 more days, until complete fixation was achieved before sectioning for gross and histological examination.

SARS-COV-2 PCR-testing. The fetal and the maternal surface were separately sampled. The fetal surface was cleaned with alcohol solution to minimize the possibility of viral RNA contamination from traces of maternal blood, amniotic fluid or other maternal fluids. A section of $1 \mathrm{~cm}$ was cut with a sterile blade from the chorionic plate including one major chorionic vessel with its luminal content. Two further cut sections were obtained from the subchorionic parenchyma and the maternal surface. The samples, denoted as fetal surface, subchorionic space and maternal surface respectively, were tested for the presence of viral RNA using the genesig Real-Time PCR COVID-19 assay (2019-nCoV), following RNA extraction with Promega's Maxwell viral nucleic acid extraction with magnetic beads.

Pathological examination was carried out with standard precautions by a standard protocol. Ten blocks of tissue were examined for each placenta. Hematoxylin-Eosin (H\&E) sections were evaluated for inflammatory lesions, chorionic villous maturation, changes of fetal or maternal malperfusion, decidual arteriopathy, and other, according to the Amsterdam consensus statement for placental lesions [6]. Immunohistochemical stains for CD3, CD4, CD8 to identify lymphocyte subpopulations, and CD138 for plasmacytes, were performed on selected sections. Clinical information on the mothers and infants was correlated with the placental findings.

\section{Results}

\section{Case 1}

A 24-year-old woman (G3P2) developed fever (Tmax 38C) and cough two days before delivery. A throat swab was examined by RT-PCR assay and confirmed SARS-CoV-2 infection. A male neonate was born at $40^{+1}$ weeks of gestation, by caesarean section due to macrosomia and estimated fetal weight on ultrasound examination $>4000 \mathrm{~g}$. There was no testing for gestational diabetes. Apgar scores were 8 and

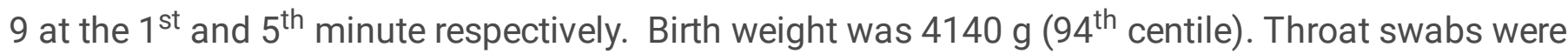
negative for SARS-CoV-2 on days 0,2 and 7 of life. He developed watery stools between the $4^{\text {th }}$ and $8^{\text {th }}$ day of life, with anal swab samples testing negative for SARS-CoV-2. The neonate had some mild problems with feeding and needed nasogastric tube feeding periodically. His clinical course and laboratory exams were otherwise unremarkable. Maternal throat swab qRT-PCR was negative during the $7^{\text {th }}$ and $8^{\text {th }}$ postpartum day, and both mother and baby were discharged in good health.

Placenta (P1): The placenta trimmed weight $-410 \mathrm{~g}$ fell between the $10^{\text {th }}$ and $25^{\text {th }}$ centile. There were no conspicuous gross abnormalities on inspection and sectioning of the placenta. On microscopy, the umbilical cord showed mild aggregates of neutrophils marginating in the umbilical vein, without penetration of the vascular wall or the surrounding Wharton jelly. The endothelium of the umbilical 
vessels at sites appeared vacuolated (Figure 1A). In the fetal vasculature, some stem villous vessels showed intraluminal inflammatory aggregates of mixed type, with recent mural thrombus formation (Figure 1B) not associated with downstream avascular villi. Sparse isolated intraluminal CD4+ or CD8+ Tlymphocytes were noted in the distal villous vessels. The chorionic villi showed a discordant development, with areas of retarded maturation, and an increase in syncytial knots over one third of the examined parenchyma. There were various degrees of subchorionic and intervillous fibrin/fibrinoid deposition, at sites showing degradation (Figure 2C), or associated with increased shedding of the perivillous trophoblast. Sparse inflammatory cells of mixed type, including macrophages, were seen within the intervillous fibrin. Fibrin deposition was accentuated close to the basal plate, and two microscopic infarcts were also identified. The maternal decidua showed mild multifocal infiltrates of CD3+ lymphocytes, either CD8+ or CD4+, admixed with monocytes and few plasmacytes (Figure 2D,E,F); in one single focus they appeared associated with overlying chronic basal villitis. Vacuolation of the decidual cells appeared prominent; some multinucleated syncytiotrophoblastic cells were highly vacuolated. Obliterative thrombosis was seen in two decidual vessels, surrounded by monocytes. No signs of frank decidual arteriopathy could be identified.

\section{Case 2}

A 31-years-old (G3P2) woman presented during her 38th week of gestation with sore throat, runny nose, low grade fever and cough. On her 4th day of illness, two days prior to the date of elective caesarean section due to two previous caesarean sections, a throat swab examination by RT-PCR confirmed SARSCoV-2 infection. A male neonate was born at $38^{+5}$ weeks of gestation with Apgar score of 9 at the 1 st and 5th minute. Birth weight was $3450 \mathrm{~g}$ (50th-75th percentile). Although the mother deferversced on the 3rd postpartum day, she remained SARS-CoV-2 positive on her throat's swab until discharge 30 days post delivery. Newborn's throat swabs were negative for SARS-CoV-2 by RT-PCR on day 0, 2 and 7 of life. He, as well, developed watery diarrhea on day 2 , while on day 3 he presented with a max of 38C. Full sepsis work up was performed and treatment with ampicillin and gentamicin was initiated. However, his laboratory examination was normal and all cultures were negative, therefore antibiotics were stopped two days later. Watery stools persisted for 5 days but the baby was well appearing and free of any other symptoms. He remained in NICU since weight gain was poor and needed nasogastric feedings periodically. Throat swab samples were negative for SARS-CoV-2 on day 0, 2 and 7, while stool samples tested negative on day 2, 6 and 14 of life. Due to prolonged and persistent maternal carriage, he was discharged on the 30th day of life.

Placenta (P2): The placenta trimmed weight $-407 \mathrm{~g}$ fell between the $10^{\text {th }}$ and $25^{\text {th }}$ centile. The membranes showed mild meconium staining. The placental disk was remarkable for extensive areas of remote subchorionic thrombosis and fibrin deposition, extending along the peripheral margins and occupying over $25 \%$ of the fetal surface (Figure $1 \mathrm{~A}$ ). The basal plate was noted to be thick. On histology, remote laminated subchorionic thrombosis with fibrin deposition was confirmed (Figure 2A-inset), and was associated with mild inflammatory infiltrates consisting of polymorphs and monocytes adjacent to the subchorionic fibrin. Of note, multiple particles of undetermined origin appeared to accumulate in the 
subchorionic space, admixed with maternal red blood cells in areas of fresh hemorrhage or more remote thrombosis (Figure 2B). Besides the subchorionic space, increased intervillous fibrin deposition and degradation (Figure $2 \mathrm{C}$ ) was also noted throughout the parenchyma and adjacent to the basal plate. The latter in addition showed recent and more remote basal thrombi, as well as multifocal inflammatory exudates consisting of lymphomononuclear cells, plasmacytes and macrophages, similar to P1, but more numerous (Figure 2D,E,F). Some cells showed empty nuclei with a condensed nuclear membrane, without any conspicuous viral cytopathy though. The chorionic villi were cellular and showed a discordant development with areas of retarded maturation. Syncytial knots were prominent throughout the examined sections. No changes of chronic villitis or frank intervillositis were observed. Sparse polymorphs and $\mathrm{CD} 3+/ \mathrm{CD} 8+$ or $\mathrm{CD} 3+/ \mathrm{CD} 4+$ lymphocytes were scattered within the fetal capillaries of the chorionic villi. Notably, particles as described above, were identified in two small distal fetal vessels. The cord showed segmental vesicular changes of the vascular endothelium, more conspicuous in the umbilical vein. There were no signs of fetal inflammatory reaction. In the free membranes, some aggregates of mononuclear cells were focally noted in the chorion, associated with similar changes in the underlying decidua capsularis.

q-RT-PCR test results: All the samples taken from both placentas tested negative for SARS-CoV-2 RNA.

\section{Discussion}

\section{Main findings - Tendency toward thrombosis and fibrin degeneration}

The pathological findings in these two cases were overall of a mild degree and involved the maternal more than the fetal components of the placenta. The maternal changes in P2 were more intense than P1. One remarkable finding was the extent of subchorionic thrombosis and fibrin deposition of P2. This lesion is nonspecific and generally assumed to be the result of stasis of maternal blood in the subchorionic space, which then leads to thrombosis, while massive subchorionic thrombohematomas have been related to hypercoagulability in the maternal circulation [7]. As no known thrombotic risk factors were recorded in either case, it may be suggested that the extensive subchorionic thrombosis and basal thrombi observed in P2, and the decidual vascular thrombosis in P1, may reflect a COVID-19-related tendency toward coagulopathy [8]. Elevations in fibrinogen and fibrin degradation products (D-dimers) [8] and increased formation of fibrin in the lungs [9] have also been observed in patients with COVID-19; this tendency toward fibrin formation is likely to account for the extensive subchorionic and supradecidual fibrin, as well as the patchy intervillous fibrin deposition and degradation seen in our placenta cases. Increased intervillous fibrin deposition in relation to COVID-19 has also been observed by Shanes et al. [4] and Baergen and Heller [5] in PCR-negative third trimester placentas. Moreover, this finding has been described in a small series of placentas delivered from pregnant women with severe acute respiratory syndrome caused by the SARS-COV virus during the 2003 epidemic [10]. In general, intervillous or perivillous fibrin deposition is a nonspecific finding, commonly encountered in various patterns of placental pathology, and often related to increased apoptosis or other damage of the perivillous 
trophoblast. Prominent shedding of the perivillous syncytiotrophoblast noted in our cases would support this notion, while prominent degradation of intervillous fibrin appears likely related to COVID-19.

\section{Maternal vascular malperfusion (MVM)}

Evidence of MVM included a diffuse increase in syncytial knotting and microinfarction in P1, while decidual arteriopathy was not seen in either case. MVM has also been observed in the case series reported by Shanes et al. [4] and in the case of a 32w-placenta abruption reported by Kuhrt et al. [11].

\section{Fetal vascular malperfusion (FVM)}

Concerning the fetal vasculature, frank fetal vascular malperfusion with mural thrombi in larger fetal vessels and/or focal avascular villi was seen in ten placentas in the study by Baergen and Heller [5] and in five placentas in the series presented by Shanes et al. [4]. We observed incipient mural thrombus formation in the fetal vessels of P1, however, a disorder in glucose metabolism as an underlying contributory factor could not be ruled out in this case of fetal macrosomia. On the other hand, the intraluminal inflammatory aggregates in the umbilical vein and stem villous vessels also reflected an incipient reaction and could not be interpreted as an established fetal inflammatory response, as in the case of second trimester miscarriage with funisitis [1]. These observations are in alignment with the short interval between the onset of symptoms and delivery, but underscore the potential harmful effects of COVID-19 in cases of early-onset infection during pregnancy. The vacuolated endothelium observed in the umbilical vessels of our cases might possibly be related to the endotheliotropic nature of SARS-COV-2 virus.

\section{Maternal inflammation}

Maternal decidual inflammatory changes have not been reported to date in COVID-19 placentas. Inflammatory exudates with lymphomononuclear cells consisting of T-helper and cytotoxic lymphocytes, monocytes, macrophages, and some plasmacytes were seen in both cases in the maternal decidua, and, to a milder degree, in the subchorionic space of maternal blood. Plasmacytes, responsible for antibody formation, have been related to intrauterine transplacental viral infections, such as Cytomegalovirus and others; in our COVID-19 cases they were restricted to the maternal decidua and overlying intervillous blood space, while not being detected in the chorionic villi. Monocytes and macrophages on the other hand appear to characterize the inflammatory reaction triggered by SARS-COV-2 [12]. Exudates with a predominantly lymphocytic inflammation and multinucleated giant cells have been described in the lungs of autopsied COVID-19 patients [13]. The presence of some highly vacuolated multinuclear syncytial cells seen in the maternal decidua of our placentas is of unknown significance and may be unrelated to COVID-19.

With regard to amniotic sac infection, there was no evidence of established histologic chorioamnionitis, although the presence of some clusters of monocytes in the chorionic membranes and underlying decidua capsularis may suggest an incipient maternal infiltrate of the free membranes, blood-borne or 
spread by direct extension from the decidua basalis. No evidence of frank intervillositis or villitis was seen in our two cases.

\section{Conclusions}

Placental pathology was present even in mild disease of presumably very short duration at term pregnancy. The observed changes mainly involved the maternal tissues and blood-occupying space, and appeared related to, though not pathognomonic of COVID-19. The decidual inflammatory exudates, described for the first time in COVID-19 placentas, and the tendency toward thrombosis, fibrin production and degradation, suggest the systemic nature of COVID-19, while on the other hand, fetoplacental vascular changes and endothelial vacuolation associated with mild neonatal morbidity, may suggest a possible transplacental impact on the fetus, to be further investigated. The impact of long-term gestational COVID-19, with SARS-COV-2 infection occurring early in pregnancy remains a challenge to be confronted in the immediate future.

\section{Declarations}

Compliance with Ethical Standards: Informed consent is available

Funding: none

Conflict of interest: The authors declare that they have no conflict of interest.

\section{Authors'Contribution}

Anastasia E. Konstantinidou: placenta examination and diagnosis - conceptualization, writing original draft, visualization, literature search, review, editing

Vassiliki Papaevangelou: conceptualization, writing, review, editing - supervision of data collection

Athanassios Tsakris: conceptualization, review, editing - supervision of molecular testing

Nikolaos E. Spanakis: molecular testing - writing, contribution to literature

Garyfallia Syridou: virologic studies of the newborns - critical review, contribution to literature, submission of manuscript

Alexandros Pergaris: placenta examination, sampling for PCR, visualization - writing

Helen Kapsambeli: clinical management of the newborns, clinical data collection - critical review

Vassiliki Sideri: management of patients, clinical data collection - contribution to literature, critical review

Anna Daskalaki: coordination of clinical data collection - critical review, editing 
Sophia N. Kalantaridou: conceptualization, supervision, review, editing - management of pregnant women and deliveries, coordination of clinical data collection

All authors approved the final manuscript as submitted and agree to be accountable for all aspects of the work.

\section{References}

1. Baud D, Greub G, Favre G, et al (2020) Second-Trimester Miscarriage in a Pregnant Woman with SARS-CoV-2 Infection. JAMA doi: 10.1001/jama.2020.7233.

2. Penfield CA, Brubaker SG, Limaye MA, et al (2020) Detection of SARS-COV-2 in Placental and Fetal Membrane Samples. AJOGMF 100133 PII: S2589-9333(20)30076-8. doi:10.1016/j.ajogmf.2020.100133

3. Patanè L, Morotti D, Giunta MR, et al (2020) Vertical transmission of COVID-19: SARS-CoV-2 RNA on the fetal side of the placenta in pregnancies with COVID-19 positive mothers and neonates at birth. AJOGMF 100145. doi: 10.1016/j.ajogmf.2020.100145

4. Shanes ED, Mithal LB, Otero S, Azad HA, Miller ES, and Goldstein JA (2020) Placental Pathology in COVID-19. Am J Clin Pathol doi: 10.1093/AJCP/AQAA089

5. Rebecca N Baergen and Debra S Heller (2020) Placental Pathology in Covid-19 Positive Mothers: Preliminary Findings. Pediatr Dev Pathol 23(3): 177-180. doi: 10.1177/1093526620925569

6. Khong TY, Mooney EE, Ariel I, et al (2016) Sampling and Definitions of Placental Lesions: Amsterdam Placental Workshop Group Consensus Statement. Arch Pathol Lab Med 140:698-713. doi: 10.5858/arpa.2015-0225-CC.

7. Fox H, Sebire NJ (2007) Pathology of the placenta. WB Saunders, Philadelphia; p.116-130.

8. COVID-19 and Coagulopathy. Hematology https://www.hematology.org/covid-19/covid-19-andcoagulopathy

9. Tian S, Xiong Y, Liu H, et al (2020) Pathological study of the 2019 novel coronavirus disease (COVID19) through postmortem core biopsies. Modern Pathol doi:0.1038/s41379-020-0536-x.

10. Ng WF, Wong SF, Lam A, et al (2006) The placentas of patients with severe acute respiratory syndrome: a pathophysiological evaluation. Pathology 38:210-218.

11. Kuhrt K, McMicking J, Nanda S, Nelson-Piercy C, Shennan A (2020) Placental abruption in a twin pregnancy at 32 weeks' gestation complicated by COVID-19, without vertical transmission to the babies. AJOGMFM doi: 10.1016/j.ajogmf.2020.100135

12. Merad M and Marti JC (2020) Pathological inflammation in patients with COVID-19: a key role for monocytes and macrophages. Nature Reviews. Immunology doi:0.1038/s41577-020-0331-4 .

13. Barton LM, Duval EJ, Stroberg E, Ghosh S, Mukhopadhyay S (2020) COVID-19 Autopsies, Oklahoma, USA. Am J Clin Pathol 153:725-33. doi: 10.1093/ajcp/aqaa062. 


\section{Figures}
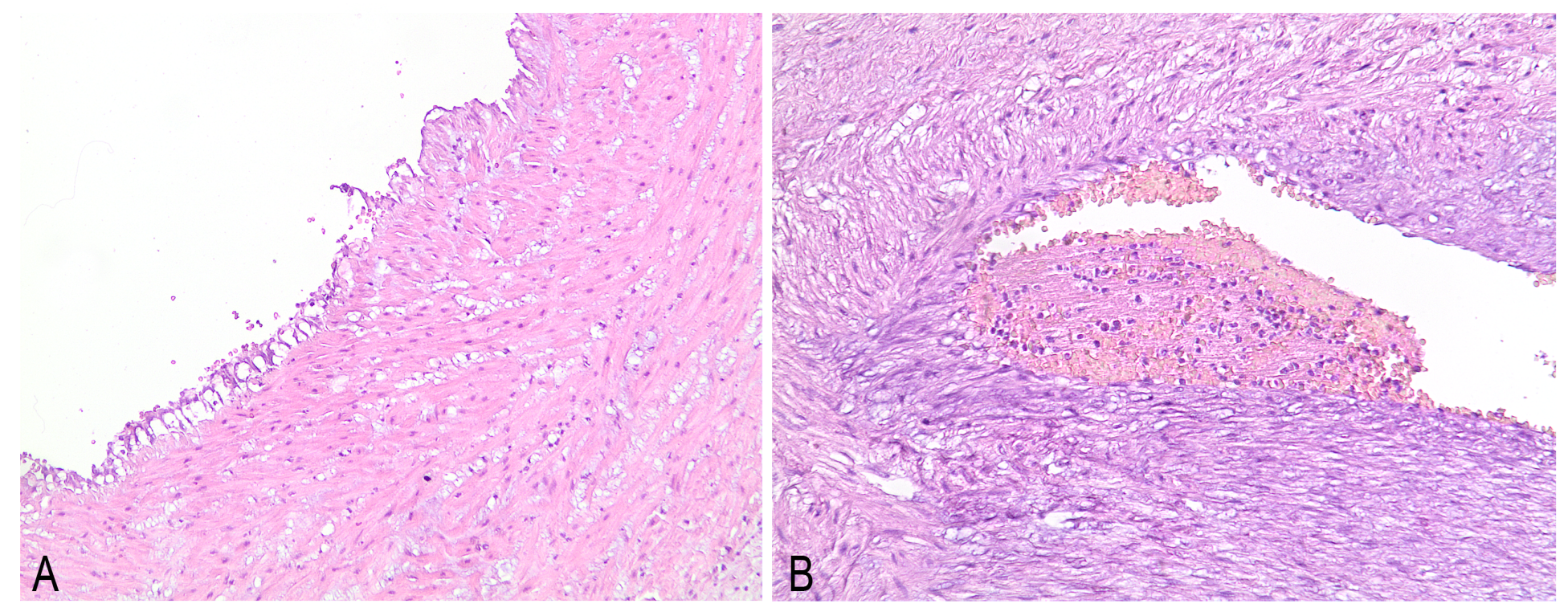

\section{Figure 1}

A: Segmental vacuolation in the endothelium of the umbilical vein $(\mathrm{H}-\mathrm{E})$. B: Fetal stem villous vessel with mural thrombus $(\mathrm{H}-\mathrm{E})$ 

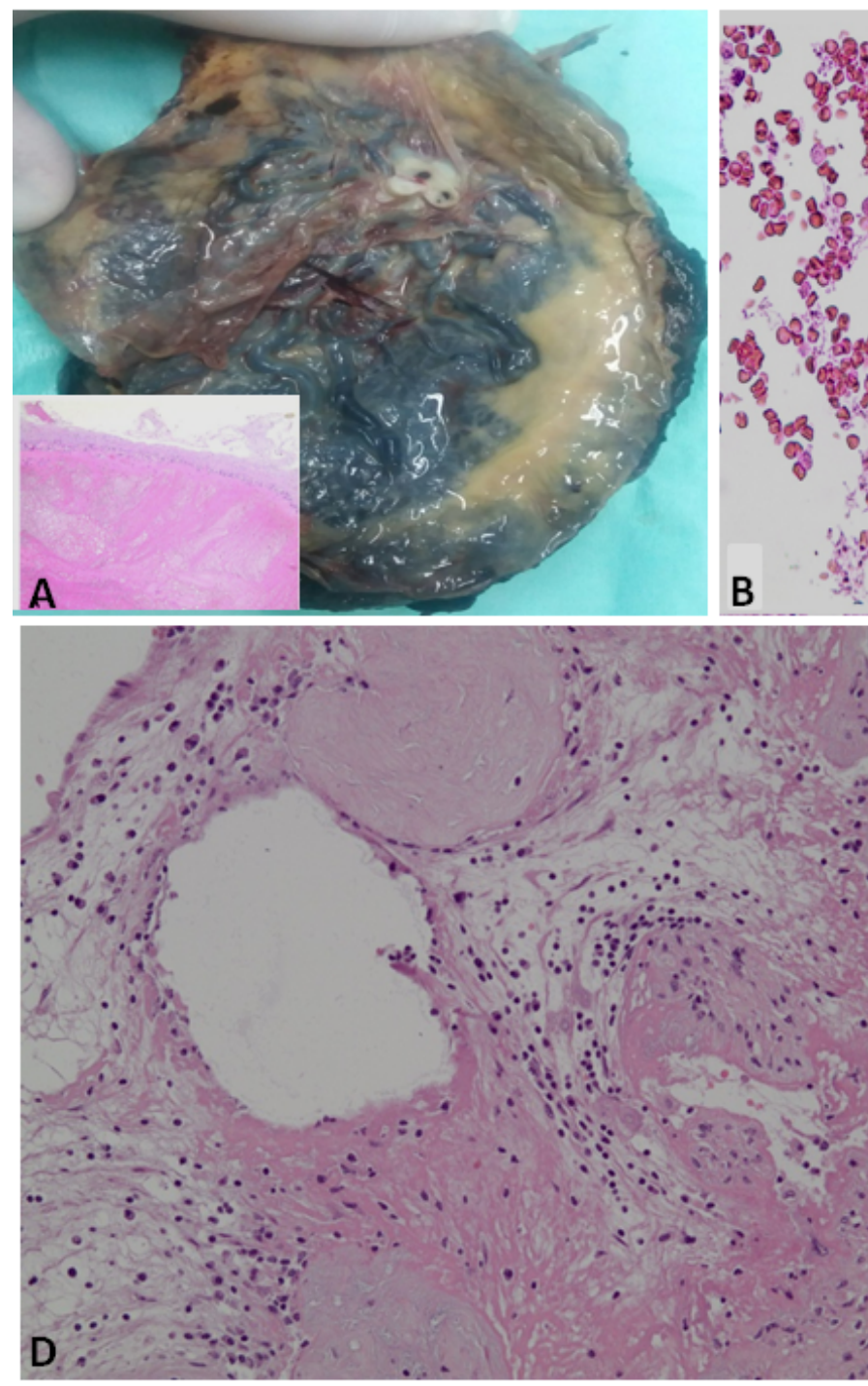
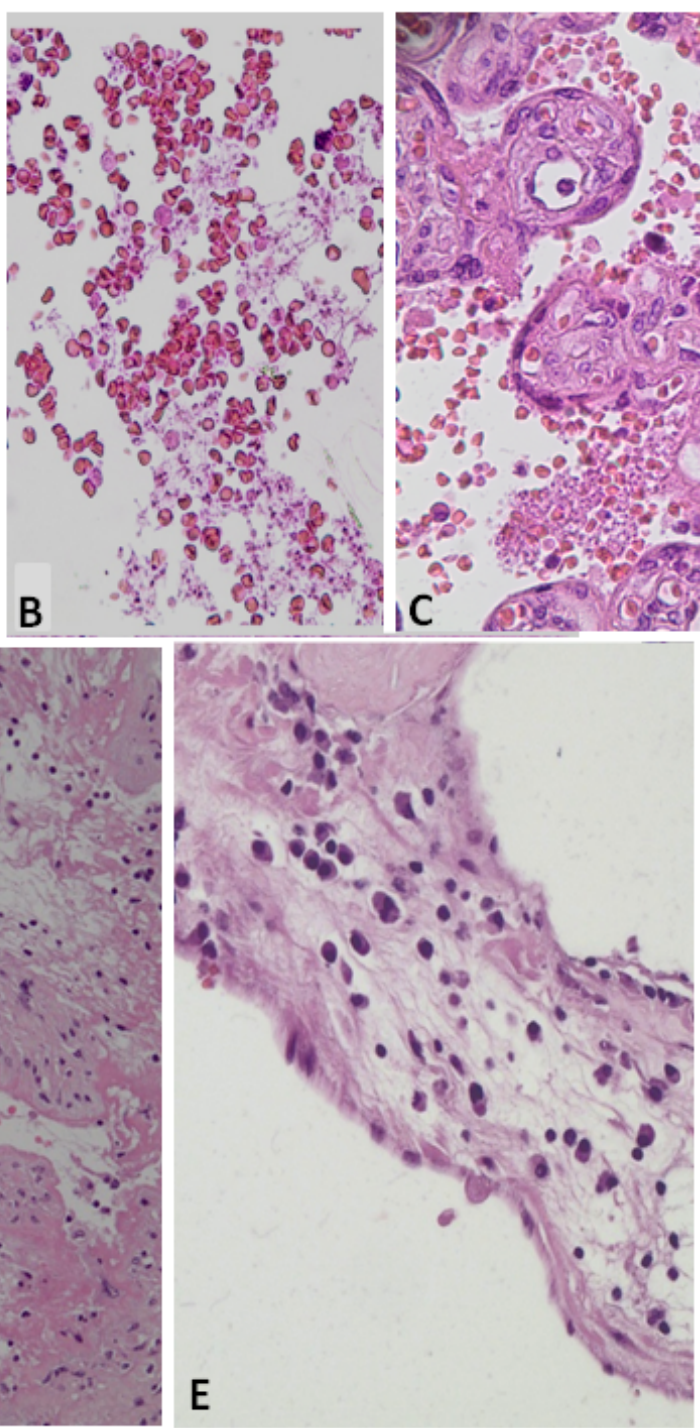
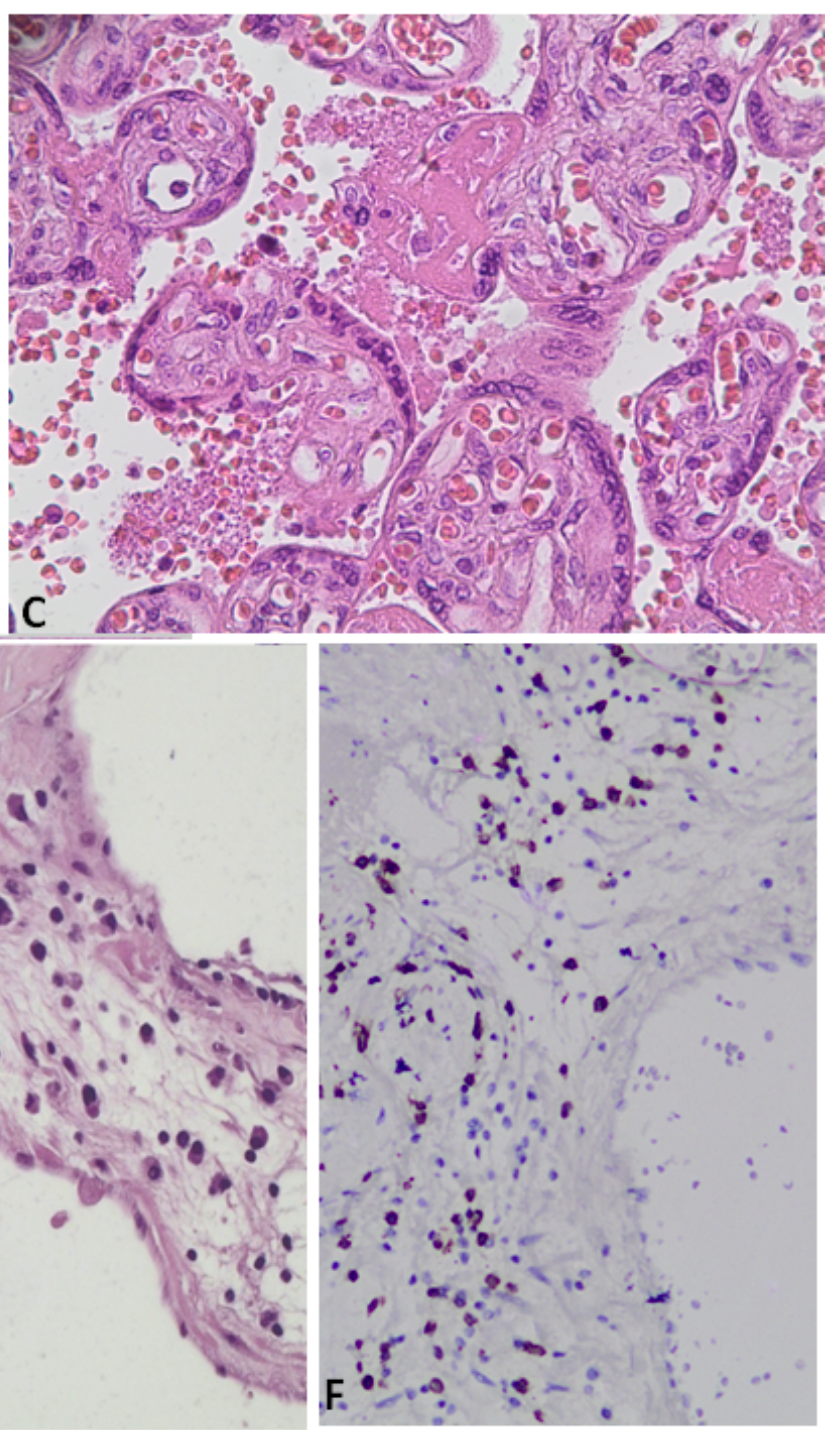

\section{Figure 2}

Macroscopic and histologic findings in Case 2. (Histology was similar but less pronounced in Case 1). A: Extensive subchorionic thrombosis confirmed on histology (inset, $\mathrm{H}-\mathrm{E}$ ). B: Particles of undetermined origin close to maternal red blood cells $(\mathrm{H}-\mathrm{E})$. C: Intervillous fibrin deposition and degradation (H-E). D, E: Decidual inflammatory exudate with mononuclear cells and plasmacytes $(\mathrm{H}-\mathrm{E})$. F: Cytotoxic lymphocytes in the exudate (CD8 immunohistochemical stain). 